\title{
Effects of different selenium sources on sow reproductive performance and piglet development: a meta-analysis
}

\author{
S.Y. Zhou ${ }^{1,2}$, B.X. Wu ${ }^{1,2}$, Z. Liu ${ }^{1,2}$ and T.J. Zhang ${ }^{1,2,3}$ \\ Yangzhou University, Yangzhou, 225009, China \\ ${ }^{1}$ College of Veterinary Medicine \\ 2 Jiangsu Co-innovation Center for Prevention and Control of Important Animal Infectious Diseases and Zoonoses
}

KEY WORDS: meta-analysis, piglets, selenium, sow reproduction

Received: 18 March 2021

Revised: $\quad 7$ May 2021

Accepted: 10 June 2021

\begin{abstract}
Selenium is an essential trace element for pig growth; generally, inorganic or organic selenium is used for dietary selenium supplementation. Newborn piglets obtain all nutrients from the sow; thus, the sow absorption rate of different selenium sources significantly affects sow reproductive performance and piglet development. Therefore, a meta-analysis was performed to provide insights into whether organic selenium is better than mineral selenium for improving sow reproductive performance and piglet development. The present study investigated the effects of different selenium sources in sow feed using articles published between June 1, 1980, and December 31, 2020, from four databases. In the meta-analysis, 19 articles (347 sows) from seven countries were included. It was shown that feeding organic selenium to sows during pregnancy significantly increased newborn piglet weight $(P<0.05)$, piglet weight at weaning $(P<0.05)$, litter weight at weaning $(P<0.01)$, selenium content in sow colostrum $(P<0.01)$, milk $(P<0.01)$ and serum $(P<0.01)$; and selenium content $(P<0.01)$ and glutathione peroxidase activity $(P<0.05)$ in piglet serum. However, it had no effect on the number of liveborn piglets and litter weight at birth. Owing to study heterogeneity $(R>50 \%)$, we hypothesized that the sow breed or weaning age were the influencing factors. Publication bias and sensitivity analyses indicate that the present results are robust. Further research is needed to better understand how different selenium sources affect sow reproduction and piglet development.
\end{abstract}

${ }^{3}$ Corresponding author: e-mail: slx@yzu.edu.cn sion of immune proteins, thereby improving immunity (Fontaine et al., 1977; Wang et al., 2018). Newborn piglets obtain selenium through colostrum and milk; selenium levels in sows and the source of dietary selenium affect the selenium status of nursing and weaned piglets (Mahan et al., 1974). Piglets are more prone to selenium deficiency than sows (Hostetler and Kincaid, 2004). The selenium levels in whole blood and milk of sows vary at different stages of pregnancy (Oikawa-Takada et al., 2005); selenium supplementation in sows during pregnancy effectively increases the selenium levels in piglets.
(Oropeza-Moe et al., 2015). In cer verse environments, selenium increases the expres- 
It improves immunity and effectively enhances piglets resistance to adverse environments (Boryaev et al., 2005). For many years, scientists have investigated the effects of different selenium sources on sow reproductive performance. Several clinical efficacy trials have been conducted and published, but the quality of reported results vary greatly. In the present study, we used meta-analysis, which facilitates drawing a conclusion through statistical analysis of published data (Gurevitch et al., 2018). We investigated the effect of different selenium sources on the reproductive performance of sows and selenium levels in piglets by assessing relevant articles using the Cochrane quality evaluation criteria (Higgins and Green, 2005) and performing a meta-analysis of studies that met the inclusion criteria, to provide more powerful clinical evidencebased medical evidence.

\section{Material and methods}

The study was conducted according to the PRISMA (preferred reporting items for metaanalyses) guidelines (Stewart et al., 2015). The PRISMA checklist was used to ensure the inclusion of relevant information in the analysis.

\section{Data source}

Four online databases were manually searched: PubMed, Science Direct, Web of Science and China Academic Journals Database (CNKI). The search was limited to articles published between June 1, 1980, and December 31, 2020. Experts and scholars in this field were contacted to find ongoing trials and collect conference proceedings.

\section{Search strategy}

Relevant articles were searched using the subject heading search terms. In Web of Science and PubMed, the following search formula was used: ('sow' [Title, Keywords, Abstract]), ('selenium' [Title, Keywords, Abstract]) and ('1980/01/1' [PDAT]: '2020/12/31' [PDAT]). In Science Direct, we used the subject terms 'sow' and 'selenium' and the web pub-date $>1980$ and pub-date $<2021$; article type was specified as 'research article', and publication titles were required to include terms related to animal feed and livestock reproduction. In CKNI, we searched for Chinese characters corresponding to sow (mu zhu) and selenium (xi); the publication period was the same as that used to search articles in English.

\section{Inclusion and exclusion criteria}

Studies were considered eligible if they met the following inclusion criteria: 1) the study was a random controlled trial (RCT) that used added organic and mineral selenium; 2) the study was a single factor experiment; 3 ) the research object was healthy sows; 4) the study included organic selenium in the experimental group and mineral selenium in the control group, and the added selenium content ranged from 0.3 to $0.5 \mathrm{mg} / \mathrm{kg}$ (Surai and Fisinin, 2016). The exclusion criteria were as follows: 1) duplicate search results; 2) the original full-text article was inaccessible; 3) the study had a small sample size; 4) the experimental data were presented as a graph, and the actual values were not available.

\section{Literature screening and data extraction}

Literature screening was performed in accordance with the pre-established inclusion and exclusion criteria; the selection and identification of articles were carried out independently by two reviewers. If the results were inconsistent, they were resolved by a third party or through discussion and negotiation.

The following data were extracted from the selected articles: authors, publication date, location of research, and study characteristics such as the total number of animals in experimental and control groups, source of selenium in experimental and control groups, weaning age, a number of births, individual and litter weight of newborn and weaned piglets, the selenium content of colostrum, milk and sow and piglet serum, and glutathione peroxidase (GSH-Px) activity in piglet serum. If the data were unclear, we attempted to contact the original author to obtain the original data. Data were reported as mean \pm standard error of mean (SEM) or standard deviation (SD); the SEM values were recalculated as SD for data standardization.

\section{Literature quality assessment}

Because animal intervention studies differ from RCTs in some aspects and the systematic evaluation of animal experimental research is not yet established, the method of systematic reviews of clinical trials of animal intervention research was modified according to the Cochrane quality evaluation criteria (Higgins and Green, 2005). A RevMan 5.3 (Cochrane, London, UK) was used for quality assessment; the results of the studies were assessed for selection bias, performance bias, detection bias and loss bias (Hooijmans et al., 2014). 


\section{Heterogeneity test and statistical analysis}

A Stata 15.0 statistical software (StataCorp LLC, College Station, TX, USA) was used for statistical analysis of the included research data. Before combining effect values, it is important to determine the inter-study heterogeneity (i.e., whether the difference between the results is due to random errors) (Higgins and Thompson, 2002). First, $I^{2}$ test and $Q$ test were used to assess whether effect sizes were heterogeneous between studies, and then the appropriate effect model was selected. For $P \geq 0.10$ and $I^{2}<50 \%$, which indicate low or no statistical heterogeneity in the effect sizes between the studies, a fixed-effects model is appropriate for analysis; for $P<0.10$ and $I^{2} \geq 50 \%$, a random-effects model is appropriate (Huedo-Medina et al., 2006), and subgroup analysis is performed on factors that cause the heterogeneity. For count data, weighted mean difference (WMD) is used as the effect scale (expressed as $95 \%$ confidence interval [CI]).

\section{Publication bias and sensitivity analysis}

Egger's test was used to estimate potential publication bias; $P \geq 0.05$ indicates a small risk of publication bias, whereas $P<0.05$ indicates potential publication bias (Egger et al., 1997). If Egger's test showed a $P<0.05$, we used the trim-and-fill method to supplement the data to verify the presence of publication bias. This method not only estimates the number of missing studies but also includes missing studies, performs a new meta-analysis, and adjusts the combined effect size of the intervention (Steichen, 2000).

Sensitivity analysis was conducted to assess the stability of the research results and to test the robustness of a pooled estimate; we evaluated the effect of each study on the pooled seroprevalence by excluding individual studies in turn. A study was deemed to have no effect if the pooled estimate excluding its data was within the $95 \%$ confidence limits (Higgins and Thompson, 2002).

\section{Results}

\section{Literature search results}

According to the search strategy, a total of 588 related articles were identified from the four databases (Web of Science $=260$, PubMed $=28$, Science Direct $=194$, CNKI $=106)$. According to the article screening process developed by Cochrane (Higgins and Green, 2005), 70 duplicate articles were excluded (PubMed $=26$, Science Direct $=36, \mathrm{CNKI}=8), 480$ articles were excluded based on the title and abstract (Web of Science $=$ 233, PubMed $=2$, Science Direct $=158, \mathrm{CNKI}=$ 87), and 19 articles were excluded according to the exclusion criteria: five English and four Chinese articles showed data duplication; in one study (Park et al., 2015) selenium supplementation of $0.6 \mathrm{mg} / \mathrm{kg}$ was used; in one study (Li et al., 2020) were used unhealthy sows; one study (Kim and Mahan, 2001) described a small sample size; the full text of three studies (Rafai and Jakab, 1999; Lampe et al., 2005; Belyaev et al., 2006) could not be obtained; and four studies (Hostetler and Kincaid, 2004; Boryaev et al., 2005; Oikawa-Takada et al., 2005; Falk et al., 2019) did not provide valid data. Finally, 19 research articles (Mahan and Kim, 1996; Mahan, 2000; Mahan and Peters, 2004; Yoon and McMillan, 2006; Zheng, 2006; Jia, 2007; Quesnel et al., 2008; Svoboda et al., 2008, 2009; Li, 2009; Yue, 2012; Lin et al., 2013; Wang and Yang, 2013; Ma et al., 2014; Szuba-Trznadel et al., 2017; Xu et al., 2017; Falk et al., 2020; Mou et al., 2020a,b) from seven countries that met the inclusion criteria were included in the meta-analysis; the method and results are shown in Figure 1.

\section{Baseline data from the included studies}

The included studies come from seven countries; most studies used Yorkshire $\times$ Landrace crossbred sows, whereas a small number of studies used local pig breeds. Xu et al. (2017) did not indicate the pig breed used. The weaning age was 14-28 days in most studies. The source of the mineral selenium in the control group was sodium selenite for most studies, except Xu et al. (2017) and Zheng (2006), who used selenium oxide and mixed selenium, respectively. The source of organic selenium in the experimental group was mostly Se-enriched yeast, Sel-Plex and hydroxy-analogue of selenomethionine; the main raw material of Sel-Plex is Se-enriched yeast. The data are shown in Table 1.

\section{Detailed data from each included study}

For the meta-analysis, we selected 10 outcome indicators: the number of live piglets born; piglet and litter weight at parturition; piglet and litter weight at weaning; selenium concentration in colostrum, milk, sow serum/plasma and piglet serum/plasma; and GSH-Px activity in piglet serum. The extracted data were standardized by using standard deviation values and consistent units. For some data that could not be obtained directly from the paper, we tried to contact the author and successfully obtained the original data for two articles (Mou et al., 2020a,b). The data are presented in Table 2. 


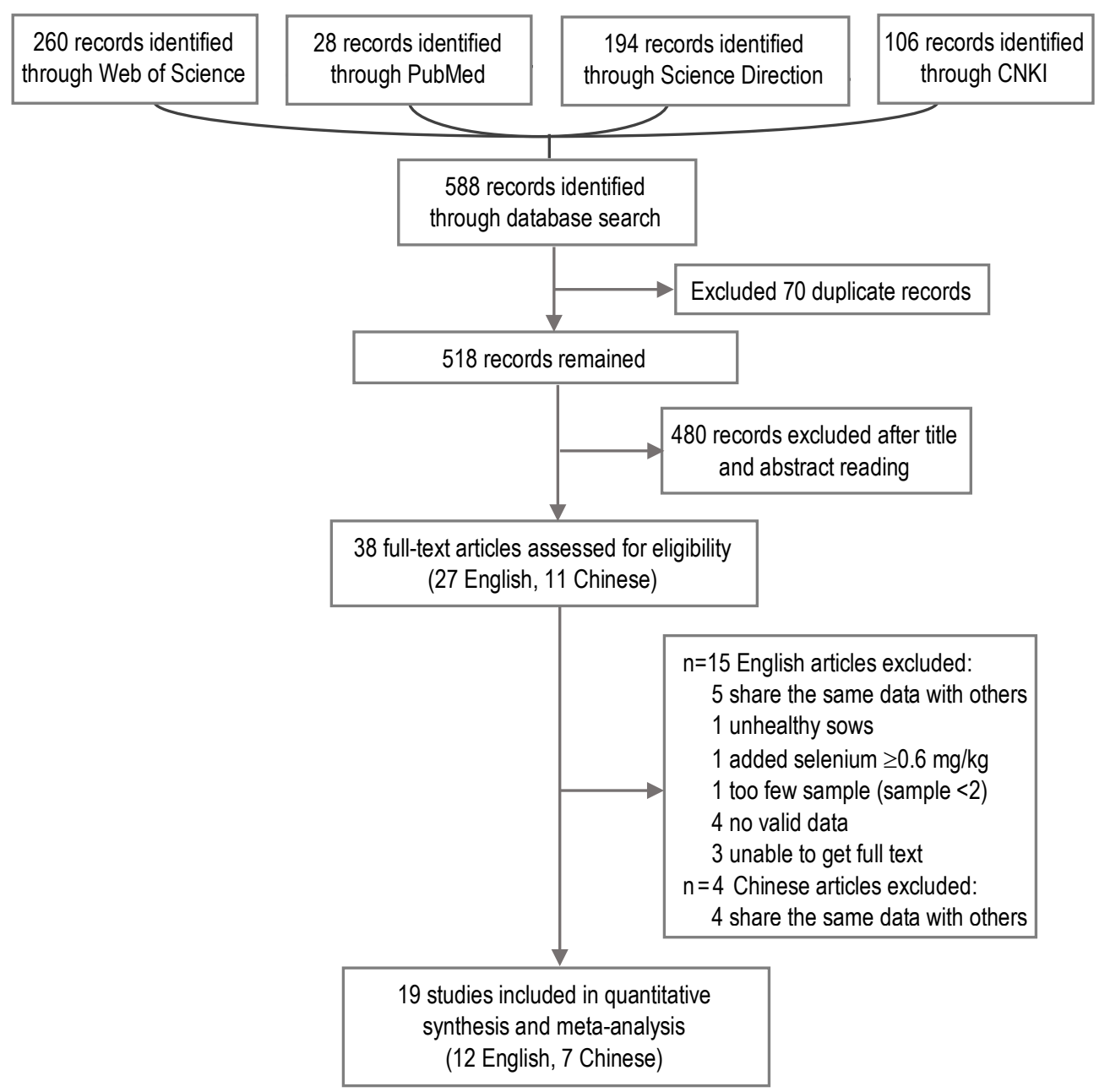

Figure 1. Flow diagram of the selection of eligible studies

Table 1. Baseline data from the studies included in the meta-analysis

\begin{tabular}{|c|c|c|c|c|}
\hline Publication & Country & Sow breed & $\begin{array}{l}\text { Weaning age, } \\
\text { day }\end{array}$ & $\begin{array}{l}\text { Selenium source, } \\
\mathrm{mg} / \mathrm{kg}\end{array}$ \\
\hline Falk et al. (2020) & Norway & Yorkshire $\times$ Landrace & 24 & SS 0.4/ L-SeMet 0.26 \\
\hline Jia (2007) & China & Yorkshire $\times$ Landrace & 21 & SS 0.3/Se-enriched yeast 0.5 \\
\hline Li (2009) & China & Yorkshire $\times$ Landrace & 28 & SS $0.3 /$ Selenomethionine 0.4 \\
\hline Lin et al. (2013) & China & Yorkshire $\times$ Landrace & 28 & SS $0.3 /$ Se-enriched yeast 0.3 \\
\hline Ma et al. (2014) & USA & Mix breed & 21 & SS 0.3/Sel-Plex 0.3 \\
\hline Mahan (2000) & USA & Yorkshire $\times$ Landrace & 14 & SS 0.3/Se-enriched yeast 0.3 \\
\hline Mahan and Kim (1996) & USA & Yorkshire $\times$ Landrace & 21 & SS $0.3 /$ Se-enriched yeast 0.3 \\
\hline Mahan and Peters (2004) & USA & Yorkshire $\times$ Landrace & $17-21$ & SS $0.3 /$ Se-enriched yeast 0.3 \\
\hline Mou et al. (2020a) & China & Yorkshire $\times$ Landrace & 21 & SS $0.3 / \mathrm{HMSeBA} 0.3$ \\
\hline Mou et al. (2020b) & China & Yorkshire $\times$ Landrace & 0 & SS 0.3/HMSeBA 0.3 \\
\hline Quesnel et al. (2008) & France & Yorkshire $\times$ Landrace & 26 & SS 0.3/Sel-Plex 0.3 \\
\hline Svoboda et al. (2009) & Czech Republic & Landrace $\times$ Czech Large White & 3 & SS 0.3/Se-enriched alga Chlorella spp. 0.3 \\
\hline Svoboda et al. (2008) & Czech Republic & Landrace $\times$ Czech Large White & 14 & SS 0.3/Sel-Plex 0.3 \\
\hline Szuba-Trznadel et al. (2017) & Poland & WBP $\times$ PBZ & 28 & SS 0.4/organic selenium 0.26 \\
\hline Wang and Yang (2013) & China & (Yorkshire $\times$ Landrace) or Tibet pig & 21 & SS 0.3/Se-enriched yeast 0.5 \\
\hline Xu (2017) & China & unknown & 21 & $\mathrm{SeO}_{2} 0.4 / \mathrm{Se}$-enriched yeast 0.3 \\
\hline Yoon and McMillan (2006) & Canada & Yorkshire $\times$ Landrace & 14 & SS $0.3 /$ Se-enriched yeast 0.3 \\
\hline Yue (2012) & China & Yorkshire $\times$ Landrace & 28 & SS $0.3 /$ Se-enriched yeast 0.3 \\
\hline Zheng (2006) & China & Yorkshire & 28 & SS $0.3 /$ SS $0.2+$ Se-enriched yeast 0.2 \\
\hline
\end{tabular}

SS - sodium selenite, SeMet - selenomethionine, Sel-Pex - Se-enriched yeast, HMSeBA - hydroxy-analogue of selenomethionine 


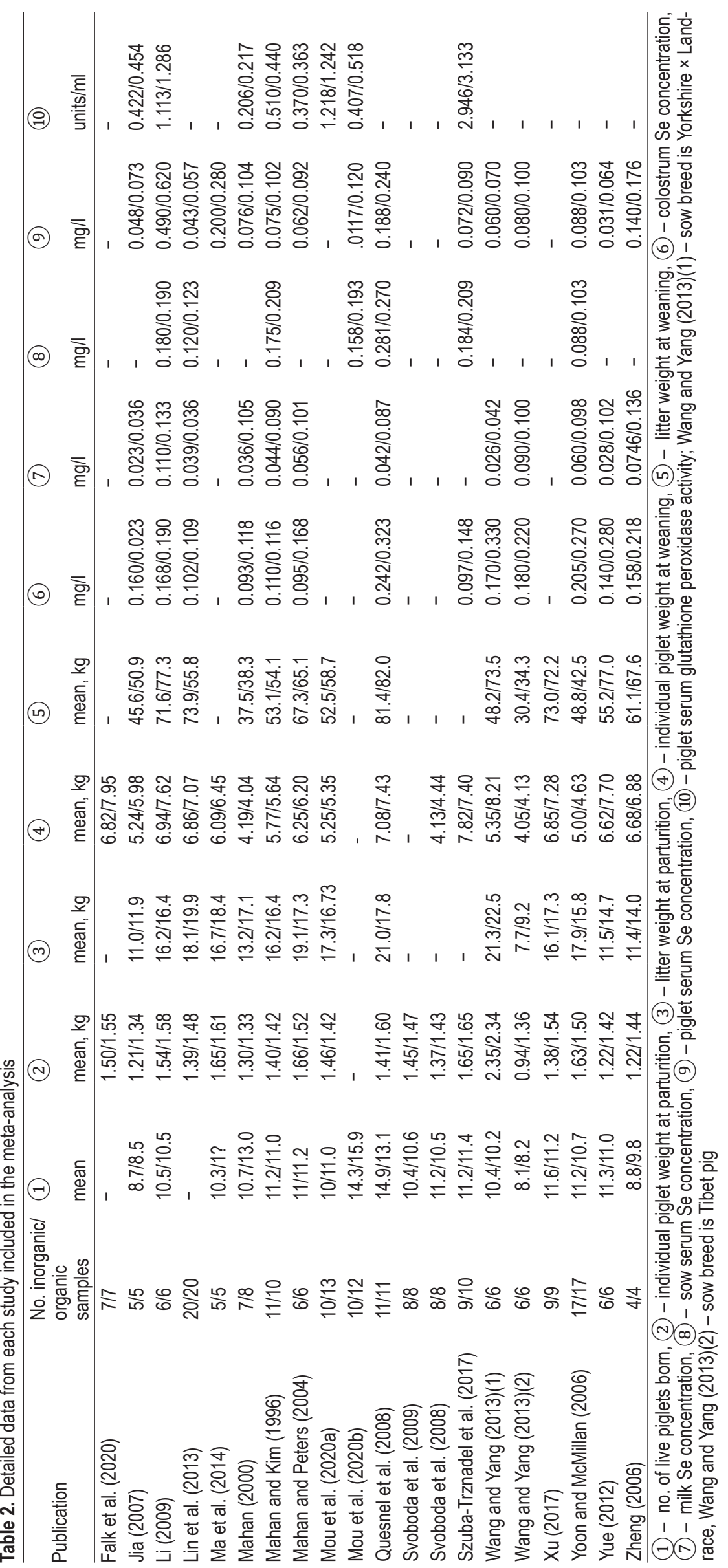




\section{Methodological quality assessment of the included studies}

Of the 19 studies, Svoboda et al. $(2008 ; 2009)$ and Szuba-Trznadel (2017) did not mention random grouping and had an uncertain risk of selection bias, whereas other studies mentioned random grouping and had a low risk of bias. In terms of baseline characteristics, only Xu et al. (2017) did not mention the sow breed and other relevant information and had a high risk of selection bias; Li (2009), Svoboda et al. (2008; 2009) and Yue (2012) did not mention random housing and had an uncertain risk of selection bias. Wang and Yang (2013) grouped the animals according to weight and had a high risk of performance bias, whereas other studies that grouped the animals randomly had a low risk of performance bias. None of the studies mentioned blinding of implementation and outcome assessment; therefore, all studies had an uncertain risk of performance bias. For random result evaluation, Zheng (2006), Jia (2007), Li (2009), Yue (2012), Lin et al. (2013), Wang and Yang (2013), Szuba-Trznadel et al. (2017) and Falk et al. (2020) did not provide the relevant information; therefore, these studies had an uncertain or low risk of detection bias. Zheng (2006), Jia (2007), Li (2009), Yue (2012), Lin et al. (2013), Wang and Yang (2013), Szuba-Trznadel et al. (2017) and $\mathrm{Xu}$ et al. (2017) did not specify the number of individuals lost to follow-up; therefore, these studies had an uncertain risk or low risk of loss bias. The results are shown in Figure 2.
We extracted data on five indicators of effects of selenium source on sow reproductive performance. Among the 19 studies, there was no statistically significant difference in the effects of feeding organic and mineral selenium on the number of live-born piglets $(\mathrm{WMD}=0.020,95 \%$ CI $[-0.561,0.600]$, $P>0.05, I^{2}=65.4 \%$ ); there was a significant difference in piglet weight at parturition $(\mathrm{WMD}=0.084,95 \%$ CI $[0.016,0.153], P<0.05$, $I^{2}=86.7 \%$ ) but no difference in piglet litter weight at parturition (WMD $=0.748,95 \%$ CI $[-0.444,1.940]$, $P>0.05, I^{2}=86.9 \%$ ) was noted. Piglet weight at weaning showed a significant increase with organic selenium supplementation $(\mathrm{WMD}=0.430,95 \% \mathrm{CI}$ [0.022, 0.873], $P<0.05, I^{2}=98.3 \%$ ), and piglet litter weight at weaning showed an extremely significant increase with organic selenium supplementation $(\mathrm{WMD}=5.386,95 \%$ CI $[1.725,9.048], P<0.01$, $I^{2}=86.4 \%$ ); Figure 3 shows a forest plot summarizing sow reproductive performance. A subgroup analysis based on sow breed showed that individual piglet weight at parturition did not differ in the Yorkshire $\times$ Landrace pigs but differed significantly in other breeds (Figure 4). A subgroup analysis based on piglet weaning age showed that the litter weight at weaning (Figure 5) was significantly higher in organic selenium groups in piglets weaned after 21 days.

In total, 12, 11 and 10 studies included data on selenium content in colostrum $(\mathrm{WMD}=0.063$, 95\% CI [0.041, 0.085], $P<0.01, I^{2}=89.2 \%$ ),

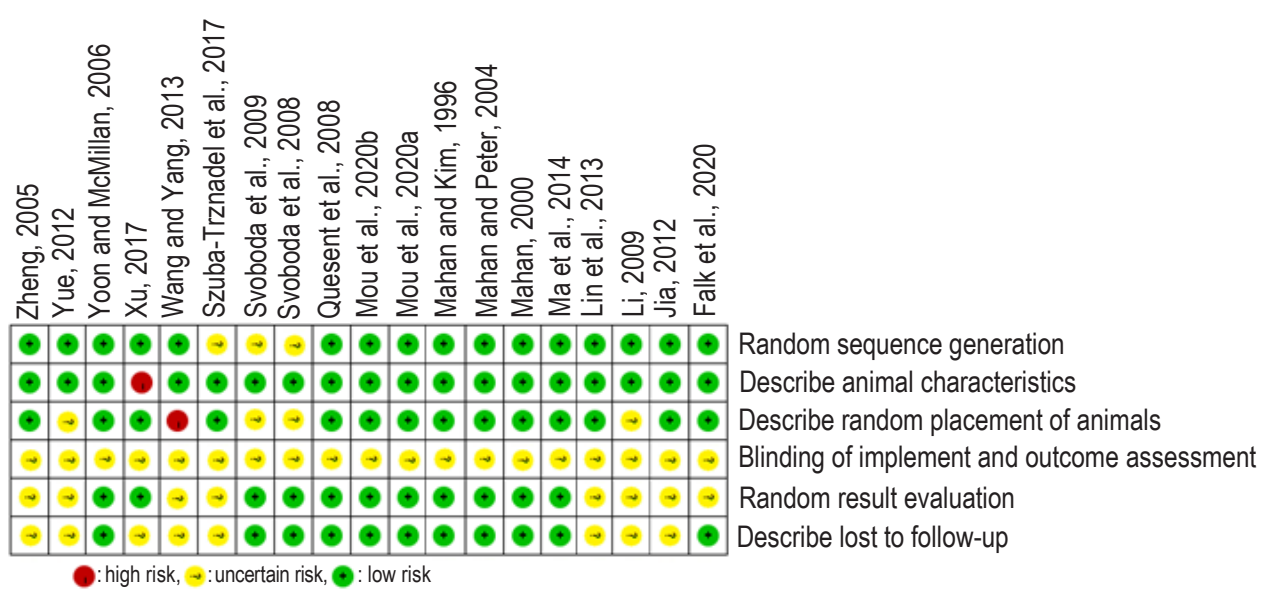

Figure 2. Bias assessment chart of eligible literature

\section{Heterogeneity test and statistical analysis}

There was substantial heterogeneity $\left(I^{2}>50 \%\right)$ among the data from the 19 articles reviewed; therefore, we used a random-effects model to combine the effect values. selenium content in milk (WMD $=0.035,95 \% \mathrm{CI}$ $\left.[0.019,0.052], P<0.01, I^{2}=99.3 \%\right)$ and selenium content in sow serum $(\mathrm{WMD}=0.010,95 \% \mathrm{CI}$ [0.003, 0.017], $P<0.01, I^{2}=90 \%$ ), respectively. There were 14 studies that mentioned the effect of 


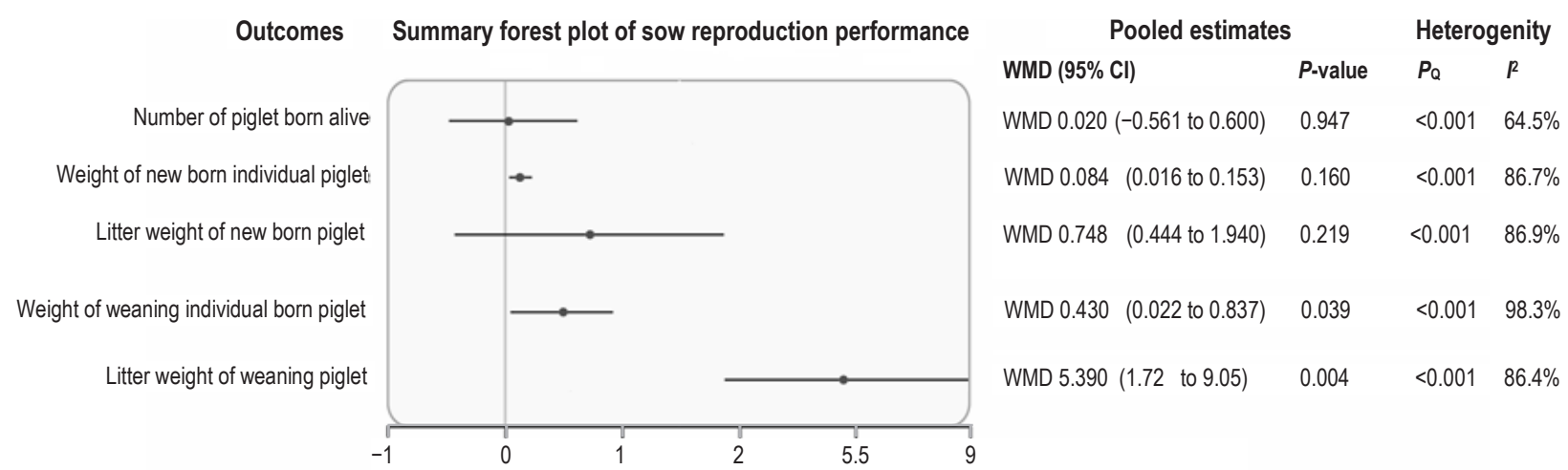

Figure 3. Forest plot of sow reproduction performance

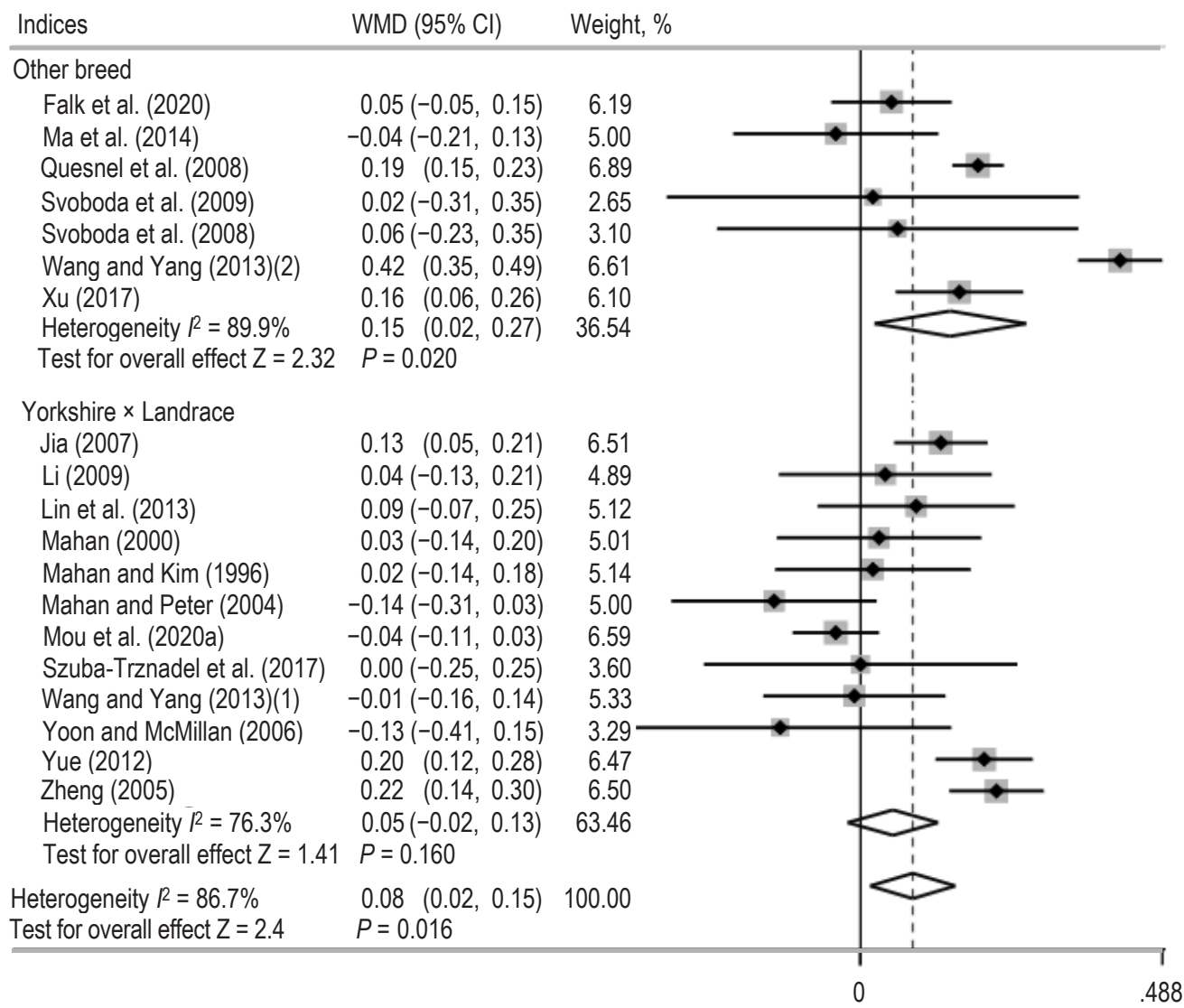

Figure 4. Forest plot of subgroup analysis of individual piglet weight at parturition on the basis of sow breed

Wang and Yang (2013)(1) - sow breed is Yorkshire $\times$ Landrace, Wang and Yang (2013)(2) - sow breed is Tibet pig

organic and mineral selenium source on selenium content in piglet serum $(\mathrm{WMD}=0.033,95 \% \mathrm{CI}$ [0.014, 0.052], $P<0.01, I^{2}=99.5 \%$ ), and eight studies measured the GSH-Px activity in piglet serum $(\mathrm{WMD}=0.044,95 \%$ CI [0.004, 0.085], $P<0.05, I^{2}=87.5 \%$ ). Figure 6 shows a forest plot summarizing the above data.

\section{Sensitivity analysis and publication bias analysis}

Sensitivity analysis was performed on the 10 indicators individually. The point effect values of all indicators fell within the $95 \%$ credible interval of the final effect value, indicating good stability and no impact on the final conclusion.

Egger's test for publication bias was performed on the 10 indicators using Stata 15.0 (StataCorp LLC, College Station, TX, USA). The results indicated that the indicator piglet weight at parturition has publication bias $(P<0.05)$; therefore, we used the trimand-fill method for this indicator. The trim-and-fill method results showed 'no trimming performed; data unchanged', and the articles included in the present paper had high-quality scores, indicating that the result is stable. The other nine indicators showed stable values $(P>0.05)$; for detailed data of each indicator. 


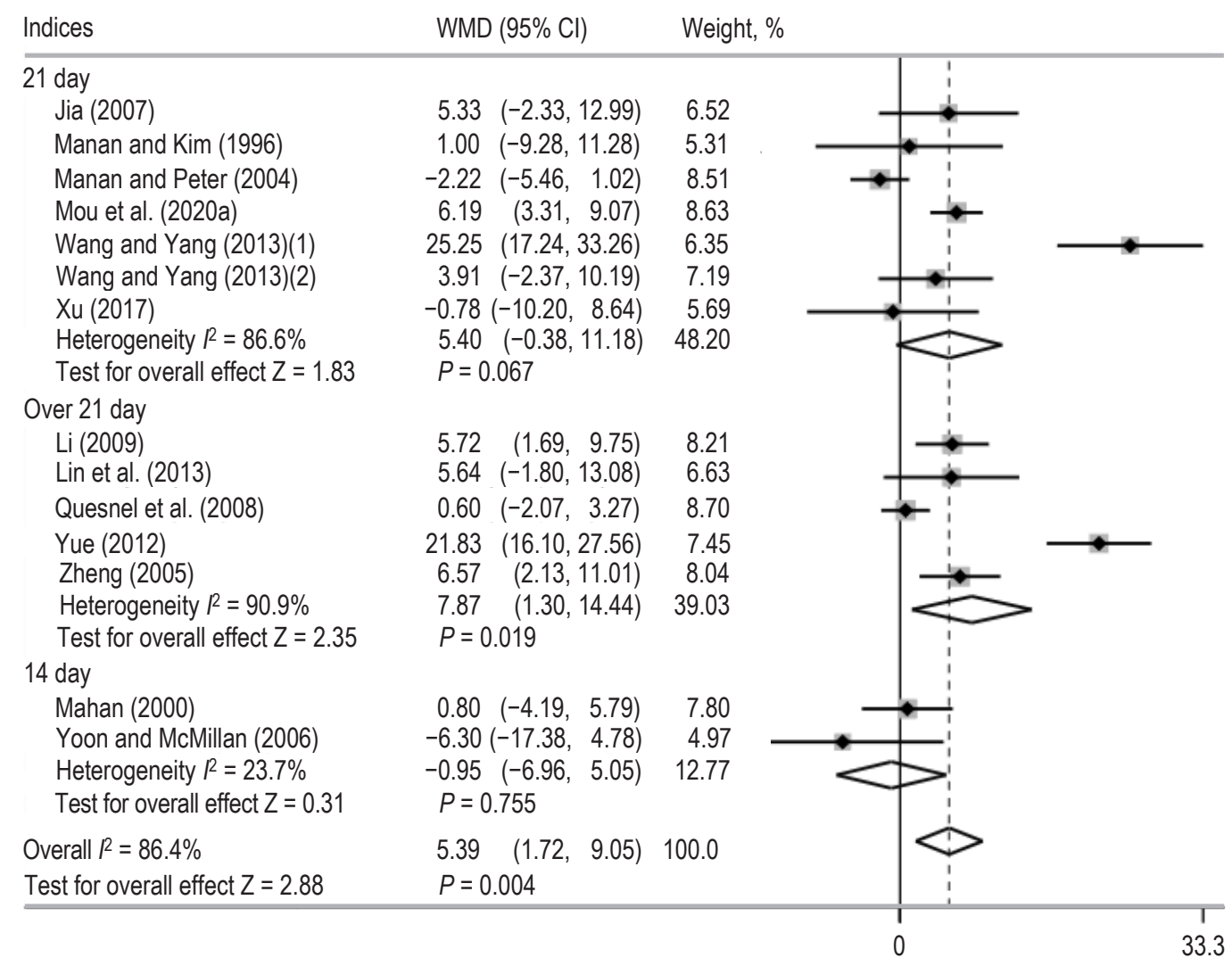

Figure 5. Forest plot of subgroup analysis of piglet litter weight at weaning on the basis of weaning age

Wang and Yang (2013)(1) - sow breed is Yorkshire $\times$ Landrace, Wang and Yang (2013)(2) - sow breed is Tibet pig

\section{Outcomes Summary forest plot of selenium content}

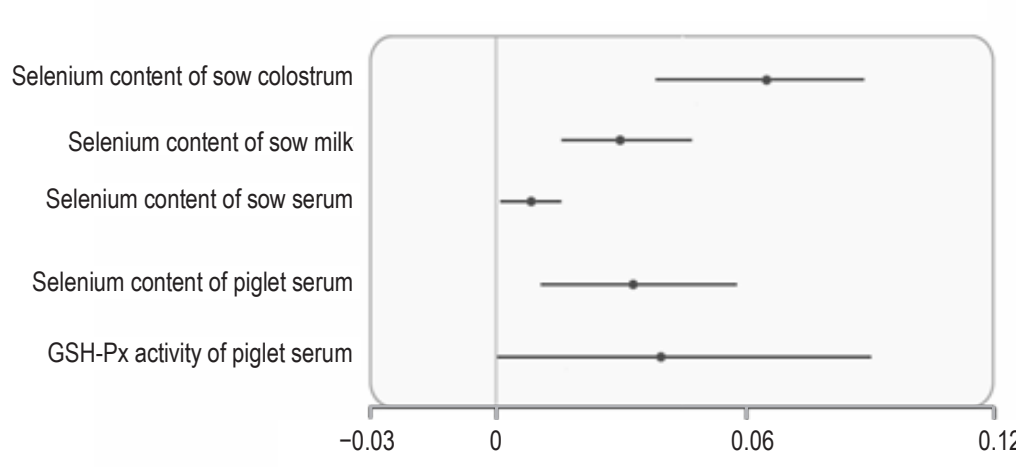

\section{Pooled estimates}

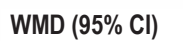

WMD 0.63 (0.041 to 0.085

WMD $0.63(0.041$ to 0.085$)$

WMD 0.035 (0.019 to 0.052$)$

WMD $0.010(0.003$ to 0.017$)$

WMD 0.033 (0.014 to 0.052$)$

WMD 0.044 (0.004 to 0.085$) \quad 0.032$
Heterogenity

$P$-value $P_{Q} \quad R$

$<0.001<0.001 \quad 89.2 \%$

$<0.001<0.001 \quad 99.3 \%$

$0.009<0.001 \quad 99.0 \%$

$0.001<0.001 \quad 99.5 \%$

$<0.001 \quad 87.5 \%$

Figure 6. Forest plot summarizing selenium content and glutathione peroxidase (GSH-Px) activity

\section{Discussion}

In recent decades, the main source of selenium in pig feed is inorganic selenium, which has a distinct limitation of high toxicity; selenium also interacts with other minerals, which cannot be effectively transferred to animals to establish and maintain selenium reserves (Surai, 2006; Surai and Fisinin, 2014). In terms of the type of organic selenium used in the included studies, most of the experiments used Se-enriched yeast. From our meta-analysis results, it can be seen that feeding organic selenium increased the selenium content in sow serum (7.7\%), colostrum (44.8\%) and milk (69.5\%). Organic selenium is more effective than inorganic one in increasing the selenium content in blood, and it persists in the animal body longer (Duntas and Benvenga, 2015). Gestation is considered to be a period of constant oxidative stress for the dam (Wisdom et al., 1991). It seems likely that at the time of gestation, sows are characterized by decreased antioxidant protection and undergo increased DNA 
damage. The reproductive performance of sows is likely associated with their oxidative stress status during gestation and lactation (Zhao et al., 2013). Serum selenium begins to decline around 60 days post coitum and decreases even more rapidly with approaching parturition. Simultaneously, GSH-Px activity also declines (Mahan et al., 2007), and increased oxidative damage in sows during late gestation may negatively affect the growth and health of foetuses and postpartum growth of piglets (Zhao et al., 2013). The selenium content of the body is also positively correlated with the production of certain antioxidants. The meta-analysis results showed that the serum selenium concentration and GSH-Px activity were 29.44 and $6.4 \%$ higher, respectively, in piglets produced by sows supplemented with organic selenium than in piglets produced by sows supplemented with inorganic selenium.

The subgroup analysis based on weaning age indicated that organic selenium effectively increased the weaning weight (by 6.93\%) of piglets, especially those weaned after 21 days, but had no significant effect on the weight of piglets weaned before 21 days; it is speculated that with prolongation of the weaning age, there is sufficient amount of selenium available to enhance the adaptability of the piglets to intestinal infections and adverse environments during the early growth process (Mahmoud and Edens, 2005; Li et al., 2020). Meta-analysis of piglet birth weight showed that individual newborn piglets produced by sows fed diets with organic selenium weighed $4.58 \%$ more than those produced by sows fed diets with inorganic selenium. Individual piglet weight significantly differed between sows fed diets with different selenium sources in pigs of other breeds but not in Yorkshire $\times$ Landrace pigs, indicating that the absorption of organic selenium varies according to breed. The difference in selenium source had no significant effect on the number of live births; a potential explanation is that the number of live births is affected more by specific genes of the parent pigs (Chen et al., 2019).

The results suggest that improvement in antioxidant activity of sows and piglets during pregnancy, lactation, and growth of piglets by feeding organic selenium is of great importance for their health and productive and reproductive performance.

In the studies included in this paper, the difference in the quantity of organic selenium and inorganic selenium added to sow feed was within $0.2 \mathrm{mg} / \mathrm{kg}$, and the total added quantity did not exceed $0.5 \mathrm{mg} / \mathrm{kg}$; however, there is still no uniform standard to determine the quantity of selenium added. We tried to choose the same dose of two different selenium sources to reduce the effect due to dose. Most of the included studies had a small sample size and therefore the metaanalysis lacked large sample data. Many of the results showed substantial and unexplainable heterogeneity. When analysing the source of the heterogeneity, due to the lack of specific information in the studies, only a few subgroup categories were available for analysis. On the basis of the results of subgroup analyses, we believe that the heterogeneity is caused by sow breed, weaning age and certain uncontrollable factors (such as environmental conditions and experimental goals). A publication bias may be caused by the lack of negative reports and samples, which is inevitable and unexplainable. Moreover, in the present metaanalysis, owing to the limited number of articles, we did not analyse other antioxidant indicators in piglet serum and immune indicators such as immunoglobulin $\mathrm{G}(\operatorname{IgG})$, and the effect of various organic selenium sources on the performance of sows and piglets should be analysed in detail. Organic selenium can increase the production of corresponding antioxidants, thereby increasing the levels of certain immune factors and improving the resistance to infectious agents (Wang et al., 2013); therefore, further research should focus on subgroup analyses to identify the potential differences in these parameters. If the results were inconsistent, they were resolved by a third party or through discussion and negotiation.

\section{Conclusions}

This meta-analysis suggests that organic selenium significantly increased the individual piglet weight at parturition and weaning; piglet litter weight at weaning; selenium content in sow colostrum, milk and serum; and selenium content and glutathione peroxidase (GSH-Px) activity in piglet serum. It may therefore be suggested that organic selenium might be recommended in the sow's nutrition; however, further research is needed to better understand how different selenium sources affect sow reproduction and piglet development.

\section{Founding}

A project was funded by the Priority Academic Program Development of Jiangsu Higher Education Institutions. 


\section{Conflict of interest}

The authors declare that there is no conflict of interest.

\section{References}

Belyaev V.I., Mel'nikova T.E., Shushchlebin V.I., 2006. Biochemical characteristics of blood in pregnant sows and their progeny under the influence of selekor. S-kh. Biol. 2, 93-94

Boryaev G., Fedorov Y., Nevitov M., 2005. About an effect of selenium preparation on immune system of young pigs. Agric. Biol. 4, 64-68

Chen Z., Ye S., Teng J., Diao S., Yuan X., Chen Z., Zhang H., Li J., Zhang Z., 2019. Genome-wide association studies for the number of animals born alive and dead in duroc pigs. Theriogenology 139, 36-42, https://doi.org/10.1016/j. theriogenology.2019.07.013

Dalgaard T.S., Briens M., Engberg R.M., Lauridsen C., 2018. The influence of selenium and selenoproteins on immune responses of poultry and pigs. Anim. Feed Sci. Technol. 238, 73-83, https://doi.org/10.1016/j.anifeedsci.2018.01.020

Duntas L.H., Benvenga S., 2015. Selenium: an element for life. Endocrine 48, 756-775, https://doi.org/10.1007/s12020-0140477-6

Egger M., Smith G.D., Schneider M., Minder C., 1997. Bias in metaanalysis detected by a simple, graphical test. Br. Med. J. 315, 629-634, https://doi.org/10.1136/bmj.315.7109.629

Falk M., Bernhoft A., Reinoso-Maset E., Salbu B., Lebed P., Framstad T., Fuhrmann H., Oropeza-Moe M., 2020. Beneficial antioxidant status of piglets from sows fed selenomethionine compared with piglets from sows fed sodium selenite. J. Trace Elem. Med. Biol. 58, 126439, https://doi.org/10.1016/j. jtemb.2019.126439

Falk M., Lebed P., Bernhoft A., Framstad T., Kristoffersen A.B., Salbu B., Oropeza-Moe M., 2019. Effects of sodium selenite and L-selenomethionine on feed intake, clinically relevant blood parameters and selenium species in plasma, colostrum and milk from high-yielding sows. J. Trace Elem. Med. Biol. 52, 176-185, https://doi.org/10.1016/j.jtemb.2018.12.009.

Fontaine M., Valli V.E., Young L.G., Lumsden J.H., 1977. Studies on vitamin $E$ and selenium deficiency in young pigs. I. Hematological and biochemical changes. Can. J. Comp. Med. 41, 41-51

Gurevitch J., Koricheva J., Nakagawa S., Stewart G., 2018. Metaanalysis and the science of research synthesis. Nature 555 , 175-182, https://doi.org/10.1038/nature25753

Hostetler C.E., Kincaid R.L., 2004. Gestational changes in concentrations of selenium and zinc in the porcine fetus and the effects of maternal intake of selenium. Biol. Trace Elem. Res. 97, 57-70, https://doi.org/10.1385/BTER:97:1:57

Higgins J.P.T., Thompson S.G., 2002. Quatifying heterogeneity in a meta-analysis. Stat. Med. 21, 1539-1558, https://doi. org/10.1002/sim.1186

Higgins J.P.T., Green S. (Editors). 2005. Cochrane Handbook for Systematic Reviews of Interventions version 4.2.5. John Wiley \& Sons. Chichester (UK)

Hooijmans C.R., Rovers M.M., de Vries R.B.M., Leenaars M., RitskesHoitinga M., Langendam M.W., 2014. SYRCLE's risk of bias tool for animal studies. BMC Med. Res. Methodol. 14, 43, https://doi.org/10.1186/1471-2288-14-43
Huedo-Medina T.B., Sánchez-Meca J., Marín-Martínez F., Botella J., 2006. Assessing heterogeneity in meta-analysis: $Q$ statistic or $l^{2}$ index? Psychol. Method. 11, 193-206, https://doi. org/10.1037/1082-989X.11.2.193

Jia J.Y., 2007. Effect of different source selenium on piglets of sows tissue selenium deposition and blood biochemical parameters. MSc Thesis. Sichuan Agriculture University. Ya'an, Sichuan (China)

Kim Y.Y., Mahan D.C., 2001. Prolonged feeding of high dietary levels of organic and inorganic selenium to gilts from $25 \mathrm{~kg}$ body weight through one parity. J. Anim. Sci. 79, 956-966, https:// doi.org/10.2527/2001.794956x

Lampe J., Gourley G., Sparks J., Stumpf T., 2005. Prewean piglet survivability: Sel-Plex verses sodium selenite as selenium source in sow diets. J. Anim. Sci. 83, Suppl. 2, 51 (abstract 205)

Li N.Y., Sun Z.J., Ansari A.R. et al., 2020. Impact of maternal selenium supplementation from late gestation and lactation on piglet immune function. Biol. Trace Elem. Res. 194, 159-167, https://doi.org/10.1007/s12011-019-01754-y

Li X., 2009. Effect of sows supplement different selenium sources on growth performance of nursing pigs and approaching to its mechanism. MSc Thesis. Zhejiang University. Zhejiang (China)

Lin C.G., Lin J.Y., Lin Z.Y., Liu Y.X., Zheng J.G., 2013. Effects of different selenium sources on the performance of sows during lactation and the selenium content in plasma and milk. Chin. J. Anim. Husb. 2013(21), 48-52, https://kns.cnki.net/ $\mathrm{kcms} /$ detail/detail.aspx?dbcode=CJFD\&filename=ZGXM201 321013\&dbname=CJFD2013

Ma Y.L., Lindemann M.D., Pierce J.L., Unrine J.M., Cromwell G.L., 2014. Effect of inorganic or organic selenium supplementation on reproductive performance and tissue trace mineral concentrations in gravid first-parity gilts, fetuses, and nursing piglets. J. Anim. Sci. 92, 5540-5550, https://doi.org/10.2527/ jas.2014-7590

Mahan D.C., 2000. Effect of organic and inorganic selenium sources and levels on sow colostrum and milk selenium content. J. Anim. Sci. 78, 100-105, https://doi.org/10.2527/2000.781100x

Mahan D.C., Kim Y.Y., 1996. Effect of inorganic or organic selenium at two dietary levels on reproductive performance and tissue selenium concentrations in first-parity gilts and their progeny. J. Anim. Sci. 74, 2711-2718, https://doi. org/10.2527/1996.74112711x

Mahan D.C., Penhale L.H., Cline J.H., Moxon A.L., Fetter A.W., Yarrington J.T., 1974. Efficacy of supplemental selenium in reproductive diets on sow and progeny performance. J. Anim. Sci. 39, 536-543, https://doi.org/10.2527/jas1974.393536x

Mahan D.C., Peters J.C., 2004. Long-term effects of dietary organic and inorganic selenium sources and levels on reproducing sows and their progeny. J. Anim. Sci. 82, 1343-1358, https:// doi.org/10.2527/2004.8251343x

Mahan D.C., Peters J.C., Hill G.M., 2007. Are antioxidants associated with pig and sow mortalities? In: Swine Nutrition Conference Proceedings. Indianapolis, IN (USA), pp. 13-21

Mahmoud K.Z., Edens F.W., 2005. Influence of organic selenium on hsp70 response of heat-stressed and enteropathogenic Escherichia coli-challenged broiler chickens (Gallus gallus). Comp. Biochem. Physiol. Part C Toxicol. Pharmacol. 141, 69-75, https://doi.org/10.1016/j.cca.2005.05.005

Mehdi Y., Hornick J.-L., Istasse L., Dufrasne I., 2013. Selenium in the environment, metabolism and involvement in body functions. Molecules 18, 3292-3311, https://doi.org/10.3390/ molecules 18033292 
Mou D., Ding D., Li S. et al., 2020a. Effect of maternal organic selenium supplementation during pregnancy on sow reproductive performance and long-term effect on their progeny. J. Anim. Sci. 98, skaa366, https://doi.org/10.1093/jas/skaa366

Mou D., Ding D., Yan H. et al., 2020b. Maternal supplementation of organic selenium during gestation improves sows and offspring antioxidant capacity and inflammatory status and promotes embryo survival. Food Funct. 11, 7748-7761, https://doi.org/10.1039/DOFO00832J

Oikawa-Takada N., Oshida T., Dohgasaki C., Sakurai M., 2005. Changes of the selenium content in whole blood and milk from brood sows and piglets. Jpn. J. Swine Sci. 42(3), 121129, https://doi.org/10.5938/youton.42.121

Oropeza-Moe M., Wisløff H., Bernhoft A., 2015. Selenium deficiency associated porcine and human cardiomyopathies. J. Trace Elem. Med. Biol. 31, 148-156, https://doi.org/10.1016/j. jtemb.2014.09.011

Park M., Lee S.H., Pak Y.N., 2015. Analysis of selenoaminoacids and selenoproteins in blood serum of sows fed by selenium fortified feed. Anal. Sci. Technol. 28, 196-203, https://doi. org/10.5806/AST.2015.28.3.196

Quesnel H., Renaudin A., Le Floc'h N., Jondreville C., Père M.C., Taylor-Pickard J.A., Le Dividich J., 2008. Effect of organic and inorganic selenium sources in sow diets on colostrum production and piglet response to a poor sanitary environment after weaning. Animal 2, 859-866, https://doi.org/10.1017/ S1751731108001869

Rafai P., Jakab L., 1999. The effect of feed supplementation with organically bound selenium on reproductive performance of gilts and multiparous sows. Magyar Allatorvosok Lapja 121, 610-615

Stewart L.A., Clarke M., Rovers M., Riley R.D., Simmonds M., Steward G., Tierney J.F., PRISMA-IPD Development Group, 2015. Preferred reporting items for a systematic review and meta-analyses of individual participant data: the PRISMA-IPD Statement. JAMA 313, 1657-1665, https://doi.org/10.1001/ jama.2015.3656

Surai P.F., 2006. Selenium in Nutrition and Health. University of Nottingham. Nottingham (UK)

Surai P.F., Fisinin V.I., 2014. Selenium in poultry breeder nutrition: an update. Anim. Feed Sci. Technol. 191, 1-15, https://doi. org/10.1016/j.anifeedsci.2014.02.005

Surai P.F., Fisinin V.I., 2016. Selenium in sow nutrition. Anim. Feed Sci. Technol. 211, 18-30, https://doi.org/10.1016/j. anifeedsci.2015.11.006

Svoboda M., Ficek R., Drábek J., 2008. Efficacy of organic selenium from Se-enriched yeast on selenium transfer from sows to piglets. Acta Vet. Brno 77, 515-521, https://doi.org/10.2754/ avb200877040515
Svoboda M., Kotrbáček V. Ficek R., Drábek J., 2009. Effect of organic selenium from Se-enriched alga (Chlorella spp.) on selenium transfer from sows to their progeny. Acta Vet. Brno 78, 373-377, https://doi.org/10.2754/avb200978030373

Szuba-Trznadel A., Hikawczuk T., Ciura A., Fuchs B., 2017. Effect of organic and mineral selenium sources on production performance and selected physiological indicators in swine. Med. Wet. 73, 756-763, https://doi.org/10.21521/mw.5824

Steichen T.J., 2000. sbe39: Nonparametric trim and fill analysis of publication bias in meta-analysis. Stata Tech. Bull. 57, 8-14. Reprinted in Stata Tech. Bull. Reprints 10, 108-117. Stata Press. College Station, TX (USA)

Wang H., Chan Y.L., Li T.L. et al., 2013. Reduction of splenic immunosuppressive cells and enhancement of anti-tumor immunity by synergy of fish oil and selenium yeast. PLOS ONE 8, e52912, https://doi.org/10.1371/journal.pone.0052912

Wang X., Zuo Z., Deng J. et al., 2018. Protective role of selenium in immune-relevant cytokine and immunoglobulin production by piglet splenic lymphocytes exposed to deoxynivalenol. Biol. Trace Elem. Res. 184, 83-91, https://doi.org/10.1007/ s12011-017-1160-6

Wang Y.P., Yang P.B., 2013. Effect of selenium yeast, inorganic selenium on the reproduction performance of sows and the selenium in deposition in tissues of piglet. Chin. Anim. Husb. Vet. Med. 40, 161-164

Wisdom S.J., Wilson R., McKillop J.H., Walker J.J., 1991. Antioxidant systems in normal pregnancy and in pregnancy-inducted hypertension. Am. J. Obstet. Gynecol. 165, 1701-1704, https://doi.org/10.1016/0002-9378(91)90018-M

Xu G.X., Lu L.Z., Duan S.X., Chen T., Yu D.L., 2017. Effect of different levels of selenium source on growth performance, reproductive performance and hematological indexes of pregnancy sows. Feed Ind. 2017(12), 16-20, https://doi. org/10.13302/j.cnki.fi.2017.12.004

Yoon I., McMillan E., 2006. Comparative effects of organic and inorganic selenium on selenium transfer from sows to nursing pigs. J. Anim. Sci. 84, 1729-1733, https://doi.org/10.2527/ jas.2005-311

Yue Z.H., 2012. Effects of selenium from different sources on reproductive performance of sows. Chin. Swine Ind. 2012(12), 49-51, https://doi.org/10.16174/j.cnki.115435.2012.10.027

Zhao Y., Flowers W.L., Saraiva A., Yeum K.J., Kim S.W., 2013. Effect of social ranks and gestation housing systems on oxidative stress status, reproductive performance, and immune status of sows. J. Anim. Sci. 91, 5848-5858, https://doi.org/10.2527/ jas.2013-6388

Zheng J.J., 2006. Effect of feed yeast and selenium yeast on sow reproductive performance and piglet performance. MSc Thesis. Sichuan Agriculture University. Ya'an, Sichuan (China) 